\title{
Nontrivial Solution for a Nonlocal Elliptic Transmission Problem in Variable Exponent Sobolev Spaces
}

\author{
Bilal Cekic and Rabil A. Mashiyev
}

Department of Mathematics, Dicle University, 21280 Diyarbakir, Turkey

Correspondence should be addressed to Bilal Cekic, bilalcekic@gmail.com

Received 5 May 2010; Accepted 19 December 2010

Academic Editor: Stephen Clark

Copyright (C) 2010 B. Cekic and R. A. Mashiyev. This is an open access article distributed under the Creative Commons Attribution License, which permits unrestricted use, distribution, and reproduction in any medium, provided the original work is properly cited.

In this paper, by means of adequate variational techniques and the theory of the variable exponent Sobolev spaces, we show the existence of nontrivial solution for a transmission problem given by a system of two nonlinear elliptic equations of $p(x)$-Kirchhoff type with nonstandard growth condition.

\section{Introduction}

Let $\Omega$ be smooth bounded domain of $\mathbb{R}^{n}, n \geq 2$, and let $\Omega_{1} \subset \Omega$ be a subdomain with smooth boundary $\Sigma$ satisfying $\bar{\Omega}_{1} \subset \Omega$. Writing $\Gamma=\partial \Omega$ and $\Omega_{2}=\Omega \backslash \bar{\Omega}_{1}$ we have $\Omega=\bar{\Omega}_{1} \cup \Omega_{2}$ and $\partial \Omega_{2}=\Sigma \cup \Gamma$ (Figure 1).

We are concerned with the existence of positive solutions to the following system of nonlinear elliptic equations:

$$
\begin{gathered}
-M\left(\int_{\Omega_{1}} \frac{1}{p(x)}|\nabla u|^{p(x)} d x\right) \operatorname{div}\left(|\nabla u|^{p(x)-2} \nabla u\right)=f(x, u), \quad \text { in } \Omega_{1} \\
-N\left(\int_{\Omega_{2}} \frac{1}{p(x)}|\nabla v|^{p(x)} d x\right) \operatorname{div}\left(|\nabla v|^{p(x)-2} \nabla v\right)=g(x, v), \quad \text { in } \Omega_{2}, \\
v=0, \quad \text { on } \Gamma,
\end{gathered}
$$




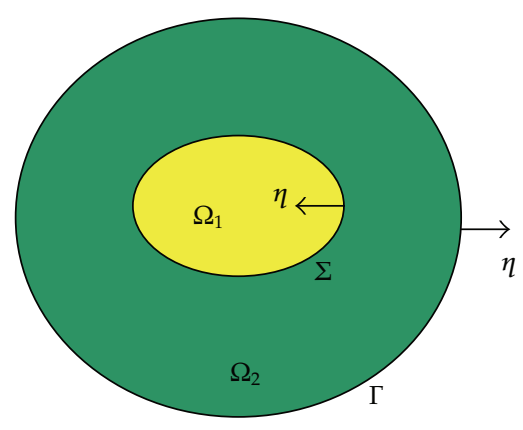

Figure 1

with the transmission conditions

$$
\begin{aligned}
M\left(\int_{\Omega_{1}}|\nabla u|^{p(x)} d x\right) \frac{\partial u}{\partial \eta} & =N\left(\int_{\Omega_{2}}|\nabla v|^{p(x)} d x\right) \frac{\partial v}{\partial \eta}, \quad \text { on } \Sigma \\
u & =v, \quad \text { on } \Sigma,
\end{aligned}
$$

where $M$ and $N$ are positive continuous functions, $\eta$ is outward normal to $\Omega_{2}$ and is inward to $\Omega_{1}$, and $p(x) \in C(\bar{\Omega})$. By means of adequate variational techniques and the theory of the variable exponent Sobolev spaces, we show the existence of nontrivial solution for a transmission problem given by a system of two nonlinear elliptic equations of $p(x)$ Kirchhoff type with nonstandard growth condition. We investigate the problem $(\mathrm{P})$ when $f(x, u)=\lambda_{1}|u|^{q(x)-2} u, g(x, v)=\lambda_{2}|v|^{q(x)-2} v$, where $\lambda_{1}, \lambda_{2}>0$ and $p(x), q(x) \in C(\bar{\Omega})$ such that $1<q(x)<p^{*}(x)$ for all $x \in \bar{\Omega}$, where $p^{*}(x)=n p(x) / n-p(x)$ if $p(x)<n$ or $p^{*}(x)=+\infty$ if $p(x) \geq n$.

The operator $-\operatorname{div}\left(|\nabla u|^{p(x)-2} \nabla u\right)$ is called the $p(x)$-Laplacian which is a natural generalization of the $p$-Laplacian when $p(x) \equiv p>1$ (a constant). The $p(x)$-Laplacian possesses more complicated nonlinearities than the $p$-Laplacian; for example, it is inhomogeneous. The study of various mathematical problems with variable exponent growth condition has received considerable attention in recent years. These problems are interesting in applications and raise many difficult mathematical problems. One of the most studied models leading to problem of this type is the model of motion of electrorheological fluids, which are characterized by their ability to drastically change the mechanical properties under the influence of an exterior electromagnetic field [1,2]. Problems with variable exponent growth conditions also appear in the mathematical modeling of stationary thermorheological viscous flows of non-Newtonian fluids and in the mathematical description of the processes filtration of an ideal barotropic gas through a porous medium [3].

Transmission problems arise in several applications in physics and biology. For instance, one of the important problems of the electrodynamics of solid media is the electromagnetic process research in ferromagnetic media with different dielectric constants. These problems appear as well as in solid mechanics if a body consists of composite materials. The existence and regularity results for linear transmission problems are well known, and a complete study can be found in [4]. We refer the reader to [5] for nonlinear elliptic transmission problems, to [6] for a nonlinear nonlocal elliptic transmission problem. 
Furthermore, uniqueness and regularity of the solutions to the thermoelastic transmission problem were investigated in [7].

We note that problem $(\mathrm{P})$ with the transmission condition is a generalization of the stationary problem of two wave equations of Kirchhoff type,

$$
\begin{aligned}
& u_{t t}-M\left(\int_{\Omega_{1}}|\nabla u|^{2} d x\right) \Delta_{2} u=f(x, u) \quad \text { in } \Omega_{1} \\
& v_{t t}-N\left(\int_{\Omega_{2}}|\nabla v|^{2} d x\right) \Delta_{2} v=g(x, v) \text { in } \Omega_{2}
\end{aligned}
$$

which models the transverse vibrations of the membrane composed by two different materials in $\Omega_{1}$ and $\Omega_{2}$. Controllability and stabilization of transmission problems for the wave equations can be found in $[8,9]$. We refer the reader to [10] for the stationary problems of Kirchhoff type, to [11] for elliptic equation $p$-Kirchhoff type, and to [12, 13] for $p(x)$ Kirchhoff type equation.

\section{Auxiliary Results}

We recall in what follows some definitions and basic properties of variable exponent Lebesgue and Sobolev spaces $L^{p(x)}(\Omega), W^{1, p(x)}(\Omega)$, and $W_{0}^{1, p(x)}(\Omega)$. In that context we refer to $[14,15]$ for the fundamental properties of these spaces.

By $\Omega$ we always denote a nonempty open subset of $\mathbb{R}^{n}$. Set

$$
C_{+}(\bar{\Omega})=\{h ; h \in C(\bar{\Omega}), h(x)>1 \forall x \in \bar{\Omega}\}
$$

For any $h \in C_{+}(\bar{\Omega})$ we define

$$
h_{1}:=\inf _{x \in \Omega} h(x), \quad h_{2}:=\sup _{x \in \Omega} h(x)
$$

We define the variable exponent Lebesgue space $L^{p(\cdot)}(\Omega)$ to consist of all measurable functions $u: \Omega \rightarrow \mathbb{R}$ for which the modular

$$
\rho_{p(x), \Omega}(u)=\int_{\Omega}|u(x)|^{p(x)} d x
$$

is finite. We define the Luxemburg norm on this space by the formula

$$
\|u\|_{p(x), \Omega}=\inf \left\{\delta>0: \rho_{p(x), \Omega}\left(\frac{u}{\delta}\right) \leq 1\right\}
$$


Equipped with this norm, $L^{p(\cdot)}(\Omega)$ is a separable and reflexive Banach space. Define the variable exponent Sobolev space $W^{1, p(x)}(\Omega)$ by

$$
W^{1, p(x)}(\Omega)=\left\{u \in L^{p(x)}(\Omega) ;|\nabla u| \in L^{p(x)}(\Omega)\right\}
$$

and the norm

$$
\|u\|_{1, p(x), \Omega}=\|u\|_{p(x), \Omega}+\|\nabla u\|_{p(x), \Omega}, \quad \forall u \in W^{1, p(x)}(\Omega)
$$

makes $W^{1, p(x)}(\Omega)$ a separable and reflexive Banach space. The space $W_{0}^{1, p(x)}(\Omega)$ is denoted by the closure of $C_{0}^{\infty}(\Omega)$ in $W^{1, p(x)}(\Omega) . W_{0}^{1, p(x)}(\Omega)$ is a separable and reflexive Banach space.

Proposition 2.1 (see $[14,15]$ ). Let $p \in C_{+}(\Omega)$. Then conjugate space of $L^{p(x)}(\Omega)$ is $L^{q(x)}(\Omega)$, where $1 / p(x)+1 / q(x)=1$. For any $u \in L^{p(x)}(\Omega)$ and $v \in L^{q(x)}(\Omega)$, one has

$$
\left|\int_{\Omega} u v d x\right| \leq 2\|u\|_{p(x), \Omega}\|v\|_{q(x), \Omega}
$$

The next proposition illuminates the close relation between the $\|\cdot\|_{p(x), \Omega}$ and the convex modular $\rho_{p(x), \Omega}$.

Proposition 2.2 (see $[14,15])$. If $u \in L^{p(x)}(\Omega)$, then one has
(i) $\|u\|_{p(x), \Omega}<1(=1 ;>1) \Leftrightarrow \rho_{p(x), \Omega}(u)<1(=1 ;>1)$,
(ii) $\|u\|_{p(x), \Omega}>1 \Rightarrow\|u\|_{p(x), \Omega}^{p_{1}} \leq \rho_{p(x), \Omega}(u) \leq\|u\|_{p(x), \Omega^{\prime}}^{p_{2}}$
(iii) $\|u\|_{p(x), \Omega}<1 \Rightarrow\|u\|_{p(x), \Omega}^{p_{2}} \leq \rho_{p(x), \Omega}(u) \leq\|u\|_{p(x), \Omega^{\prime}}^{p_{1}}$
(iv) $\|u\|_{p(x), \Omega}=a>0 \Leftrightarrow \rho_{p(x), \Omega}(u / a)=1$.

Proposition 2.3 (see $[14,15]$ ). If $u, u_{n} \in L^{p(x)}(\Omega), n=1,2, \ldots$, then the following statements are equivalent to each other:

(i) $\lim _{n \rightarrow \infty}\left\|u_{n}-u\right\|_{p(x), \Omega}=0$;

(ii) $\lim _{n \rightarrow \infty} \rho_{p(x), \Omega}\left(u_{n}-u\right)=0$;

(iii) $u_{n} \rightarrow u$ in measure in $\Omega$ and $\lim _{n \rightarrow \infty} \rho_{p(x), \Omega}\left(u_{n}\right)=\rho_{p(x), \Omega}(u)$.

Proposition 2.4 (see [14]). In $W_{0}^{1, p(x)}(\Omega)$ the Poincare inequality holds, that is, there exists a positive constant $C$ such that

$$
\|u\|_{p(x), \Omega} \leq C\|\nabla u\|_{p(x), \Omega}, \quad \forall u \in W_{0}^{1, p(x)}(\Omega)
$$

Consequently, $\|u\|_{1, p(x), \Omega}=\|\nabla u \mid\|_{p(x), \Omega}$ are equivalent norms on $W_{0}^{1, p(x)}(\Omega)$. In what follows, $W_{0}^{1, p(x)}(\Omega)$, with $p \in C_{+}(\bar{\Omega})$, will be considered as endowed with the norm $\|u\|_{1, p(x)}$. We will use $\|u\|_{1, p(x), \Omega}=\|\nabla u\|_{p(x), \Omega}$ for $u \in W_{0}^{1, p(x)}(\Omega)$ in the following discussions. 
Proposition 2.5 (see [14, 16]). Assume that $\Omega$ is bounded, the boundary of $\Omega$ possesses the cone property and $p \in C_{+}(\bar{\Omega})$. If $q \in C_{+}(\bar{\Omega})$ and $q(x)<p^{*}(x)$ for all $x \in \bar{\Omega}$ then the embedding $W^{1, p(x)}(\Omega) \hookrightarrow L^{q(x)}(\Omega)$ is the compact and continuous.

Lemma 2.6 (see [17]). Assume that $\Omega$ is bounded and has a Lipschitz boundary with the cone property and $p \in C_{+}(\bar{\Omega})$. Then there is a continuous boundary trace embedding $W^{1, p(\cdot)}(\Omega) \hookrightarrow$ $L^{p(\cdot)}(\partial \Omega)$

Lemma 2.7 (see [17]). For any $u \in W^{1, p(x)}(\Omega)$, let

$$
\|u\|_{\partial}:=\|\nabla u\|_{p(x), \Omega}+\|u\|_{p(x), \partial \Omega} .
$$

Then, $\|u\|_{\partial}$ is a norm on $W^{1, p(x)}(\Omega)$, equivalent to the standard norm of $W^{1, p(x)}(\Omega)$.

Our analysis is based on the Sobolev space

$$
E=\left\{(u, v) \in W^{1, p(x)}\left(\Omega_{1}\right) \times W_{\Gamma}^{1, p(x)}\left(\Omega_{2}\right): u=v \text { on } \Sigma\right\}
$$

where

$$
W_{\Gamma}^{1, p(x)}\left(\Omega_{2}\right)=\left\{v \in W^{1, p(x)}\left(\Omega_{2}\right): v=0 \text { on } \Gamma\right\}
$$

Then, we have following lemma that will permit the variational setting of the problem $(\mathrm{P})$.

Lemma 2.8. $E$ is a closed subspace of $W^{1, p(x)}\left(\Omega_{1}\right) \times W^{1, p(x)}\left(\Omega_{2}\right)$, and

$$
\|(u, v)\|_{E}=\|\nabla u\|_{p(x), \Omega_{1}}+\|\nabla v\|_{p(x), \Omega_{2}}
$$

defines a norm in $E$, equivalent to the standard norm of $W^{1, p(x)}\left(\Omega_{1}\right) \times W^{1, p(x)}\left(\Omega_{2}\right)$.

Proof. It is clear that (2.12) defines a seminorm. Then suppose that $\|(u, v)\|_{E}=0$. Since $\|\nabla v\|_{p(x), \Omega_{2}}$ defines a norm on $W^{1, p(x)}\left(\Omega_{2}\right)$ and Poincaré inequality holds in $W^{1, p(x)}\left(\Omega_{2}\right)$, thus $v=0$. From transmission condition, $u=v$ on $\Sigma$, and therefore $u \in W_{0}^{1, p(x)}\left(\Omega_{1}\right)$. This shows that $(u, v)=0$, and hence (2.12) defines a norm in $E$. Now, applying the trace theorem in $W^{1, p(x)}\left(\Omega_{2}\right)$, there exists $c>0$ such that for all $v \in W_{\Gamma}^{1, p(x)}\left(\Omega_{2}\right)$

$$
\|v\|_{p(x), \Sigma} \leq C\|\nabla v\|_{p(x), \Omega_{2}} .
$$

But it is known that $\|\nabla u\|_{p(x), \Omega_{1}}+\|u\|_{p(x), \Sigma}$ defines an equivalent norm in $W^{1, p(\cdot)}\left(\Omega_{1}\right)$ from Lemma 2.7. Then combining these two remarks the result follows. 


\section{Main Results}

Let us precisely describe our assumptions in order to establish the main result. The energy functional corresponding to problem $(\mathrm{P})$ is defined as $J: E \rightarrow \mathbb{R}$,

$$
\begin{aligned}
J(u, v)= & \widehat{M}\left(\int_{\Omega_{1}} \frac{|\nabla u|^{p(x)}}{p(x)} d x\right)+\widehat{N}\left(\int_{\Omega_{2}} \frac{|\nabla v|^{p(x)}}{p(x)} d x\right) \\
& -\int_{\Omega_{1}} F(x, u) d x-\int_{\Omega_{2}} G(x, v) d x
\end{aligned}
$$

where $\widehat{M}(t)=\int_{0}^{t} M(\xi) d \xi$, and $F(x, u)=\int_{0}^{u} f(x, \xi) d \xi$. It is not difficult to show that $J \in$ $C^{1}(E, \mathbb{R})$, and as a matter of fact, $J$ is of class $C^{1}$ and weakly lower semicontinuous. In particular we have, for all $(u, v),(\varphi, \psi) \in E$,

$$
\begin{aligned}
& \left\langle J^{\prime}(u, v),(\varphi, \psi)\right\rangle \\
& =M\left(\int_{\Omega_{1}} \frac{|\nabla u|^{p(x)}}{p(x)} d x\right) \int_{\Omega_{1}}|\nabla u|^{p(x)-2} \nabla u \nabla \varphi d x \\
& \quad+N\left(\int_{\Omega_{2}} \frac{|\nabla v|^{p(x)}}{p(x)} d x\right) \int_{\Omega_{2}}|\nabla v|^{p(x)-2} \nabla v \nabla \psi d x-\int_{\Omega_{1}} f(x, u) \varphi d x-\int_{\Omega_{2}} g(x, v) \psi d x
\end{aligned}
$$

We assume the following hypotheses for $M$ and $N$.

There are positive constants $M_{1}, M_{2}, N_{1}, N_{2}$, and $\alpha$ such that

$$
\begin{aligned}
& \left(E_{1}\right): M_{1} t^{\alpha-1} \leq M(t) \leq M_{2} t^{\alpha-1}, \\
& \left(E_{2}\right): N_{1} s^{\alpha-1} \leq N(s) \leq N_{2} s^{\alpha-1}
\end{aligned}
$$

for all $t, s>0$ and $\alpha \geq 1$.

Now we state our main result.

Theorem 3.1. Let us assume that $\left(E_{1}\right)$ and $\left(E_{2}\right)$ hold. If $q_{2}<\alpha p_{1}$, then the problem $(\mathrm{P})$ with the transmission condition has at least one nonnegative solution.

Lemma 3.2. There exists $\lambda^{*}>0$ such that for any $\lambda_{1}+\lambda_{2} \in\left(0, \lambda^{*}\right)$ there exist $\rho>0$ and $r>0$ such that

$$
J_{\lambda}(u, v) \geq r, \quad \forall(u, v) \in E, \text { with }\|(u, v)\|_{E}=\rho .
$$

Proof. Since $q(x)<p^{*}(x)$ for all $x \in \bar{\Omega}$ it follows that (Proposition 2.5)

$$
\begin{aligned}
& \|u\|_{q(x), \Omega_{1}} \leq C_{1}\|u\|_{1, p(x), \Omega_{1}}, \quad \forall u \in W^{1, p(x)}\left(\Omega_{1}\right) \\
& \|v\|_{q(x), \Omega_{2}} \leq C_{2}\|v\|_{1, p(x), \Omega_{2},}, \quad \forall v \in W^{1, p(x)}\left(\Omega_{2}\right) .
\end{aligned}
$$


By Lemma 2.8 we have

$$
\|u\|_{q(x), \Omega_{1}}+\|v\|_{q(x), \Omega_{2}} \leq C_{1}\|u\|_{1, p(x), \Omega_{1}}+C_{2}\|v\|_{1, p(x), \Omega_{2}} \leq C_{3}\|(u, v)\|_{E}
$$

We fix $\rho \in(0,1)$ such that $\rho<1 / C_{3}$. Then the above relation implies

$$
\|u\|_{q(x), \Omega_{1}}+\|v\|_{q(x), \Omega_{2}}<1, \quad \forall(u, v) \in E .
$$

By Proposition 2.2(ii) and 2.5 and Lemma 2.8, we deduce that

$$
\begin{aligned}
\int_{\Omega_{1}}|u|^{q(x)} d x+\int_{\Omega_{2}}|v|^{q(x)} d x & \leq\|u\|_{q(x), \Omega_{1}}^{q_{1}}+\|v\|_{q(x), \Omega_{2}}^{q_{1}} \\
& \leq\|u\|_{q(x), \Omega_{1}}+\|v\|_{q(x), \Omega_{2}} \\
& \leq C_{4}\left(\|u\|_{1, p(x), \Omega_{1}}+\|v\|_{1, p(x), \Omega_{2}}\right) \\
& \leq C_{5}\|(u, v)\|_{E} .
\end{aligned}
$$

Using $\left(E_{1}\right),\left(E_{2}\right)$, Proposition 2.2(iii), and (3.8), we obtain that for any $(u, v) \in E$ with $\|(u, v)\|_{E}<1$ the following inequalities hold true:

$$
\begin{aligned}
J_{\lambda}(u, v)= & \widehat{M}\left(\int_{\Omega_{1}} \frac{|\nabla u|^{p(x)}}{p(x)} d x\right)+\widehat{N}\left(\int_{\Omega_{2}} \frac{|\nabla v|^{p(x)}}{p(x)} d x\right) \int_{\Omega_{2}}|\nabla v|^{p(x)} d x \\
& -\lambda_{1} \int_{\Omega_{1}} \frac{|u|^{q(x)}}{q(x)} d x-\lambda_{2} \int_{\Omega_{2}} \frac{|v|^{q(x)}}{q(x)} d x \\
\geq & M_{1} \int_{0}^{\left(1 / p_{2}\right) \rho_{p(x), \Omega_{1}}(\nabla u)} t^{\alpha-1} d t+N_{1} \int_{0}^{\left(1 / p_{2}\right) \rho_{p(x), \Omega_{2}}(\nabla u)} \eta^{\alpha-1} d \eta \\
& -\frac{\lambda_{1}}{q_{1}} \int_{\Omega_{1}}|u|^{q(x)} d x-\frac{\lambda_{2}}{q_{1}} \int_{\Omega_{2}}|v|^{q(x)} d x \\
\geq & \frac{M_{1}}{\alpha}\left(\frac{1}{p_{2}} \int_{\Omega_{1}}|\nabla u|^{p(x)} d x\right)^{\alpha}+\frac{N_{1}}{\alpha}\left(\frac{1}{p_{2}} \int_{\Omega_{2}}|\nabla v|^{p(x)} d x\right)^{\alpha} \\
& -\frac{\lambda_{1}}{q_{1}} \int_{\Omega_{1}}|u|^{q(x)} d x-\frac{\lambda_{2}}{q_{1}} \int_{\Omega_{2}}|v|^{q(x)} d x \\
\geq & \frac{\min \left\{M_{1}, N_{1}\right\}}{\alpha\left(p_{2}\right)^{\alpha}}\left(\|\nabla u\|_{p(x), \Omega_{1}}^{\alpha p_{2}}+\|\nabla v\|_{p(x), \Omega_{2}}^{\alpha p_{2}}\right)-\frac{C_{5}\left(\lambda_{1}+\lambda_{2}\right)}{q_{1}}\|(u, v)\|_{E} \\
\geq & \frac{2^{1-\alpha p_{2}} \min \left\{M_{1}, N_{1}\right\}}{\alpha\left(p_{2}\right)^{\alpha}}\left(\|\nabla u\|_{p(x), \Omega_{1}}+\|\nabla v\|_{p(x), \Omega_{2}}\right)^{\alpha p_{2}}-\frac{C_{5}\left(\lambda_{1}+\lambda_{2}\right)}{q_{1}}\|(u, v)\|_{E} \\
\geq & \frac{2^{1-\alpha p_{2}} \min \left\{M_{1}, N_{1}\right\}}{\alpha\left(p_{2}\right)^{\alpha}}\|(u, v)\|_{E}^{\alpha p_{2}}-\frac{C_{5}\left(\lambda_{1}+\lambda_{2}\right)}{q_{1}}\|(u, v)\|_{E} .
\end{aligned}
$$


By the above inequality if we define

$$
\lambda^{*}=\frac{2^{1-\alpha p_{2}} \rho^{\alpha p_{2}-1} q_{1} \min \left\{M_{1}, N_{1}\right\}}{C_{5} \alpha\left(p_{2}\right)^{\alpha}}
$$

then for any $\lambda_{1}+\lambda_{2} \in\left(0, \lambda^{*}\right)$ and $(u, v) \in E$ there exists $r>0$ such that $J_{\lambda}(u, v) \geq r>0$.

The proof of Lemma 3.2 is complete.

Lemma 3.3. There exists $\varphi, \phi \in E$ such that $\varphi, \phi \geq 0$ and $\varphi, \phi \neq 0$ and $J_{\lambda}(t \varphi, t \phi)<0$ for $t>0$ small enough.

Proof. Let $\left|\Omega_{1}\right|>0$ and $\left|\Omega_{2}\right|>0$. Moreover, let choose $\varphi, \phi \in C_{0}^{\infty}(\Omega)$ and $0 \leq \varphi(x) \leq 1$ in $\Omega_{1}, 0 \leq \phi(x) \leq 1$ in $\Omega_{2}$. Then, for any $t \in(0,1)$, by $\left(E_{1}\right)$ and $\left(E_{2}\right)$ it follows

$$
\begin{aligned}
J_{\lambda}(t \varphi, t \phi)= & \widehat{M}\left(\int_{\Omega_{1}} \frac{|t \nabla \varphi|^{p(x)}}{p(x)} d x\right)+\widehat{N}\left(\int_{\Omega_{2}} \frac{|t \nabla \phi|^{p(x)}}{p(x)} d x\right) \\
& -\lambda_{1} \int_{\Omega_{1}} \frac{|t \varphi|^{q(x)}}{q(x)} d x-\lambda_{2} \int_{\Omega_{2}} \frac{|t \phi|^{q(x)}}{q(x)} d x \\
\leq & M_{2} \int_{0}^{\left(1 / p_{1}\right) \rho_{p(x), \Omega_{1}}(t \nabla \varphi)} S^{\alpha-1} d s+N_{2} \int_{0}^{\left(1 / p_{1}\right) \rho_{p(x), \Omega_{2}}(t \nabla \phi)} \eta^{\alpha-1} d \eta \\
& -\lambda_{1} \int_{\Omega_{1}} \frac{|t \varphi|^{q(x)}}{q(x)} d x-\lambda_{2} \int_{\Omega_{2}} \frac{|t \phi|^{q(x)}}{q(x)} d x \\
\leq & \frac{M_{2} t^{\alpha p_{1}}}{\alpha\left(p_{1}\right)^{\alpha}} \rho_{p(x), \Omega_{1}}^{\alpha}(\nabla \varphi)+\frac{N_{2} t^{\alpha p_{1}}}{\alpha\left(p_{1}\right)^{\alpha}} \rho_{p(x), \Omega_{2}}^{\alpha}(\nabla \phi) \\
& -\frac{\lambda_{1} t^{q_{2}}}{q_{2}} \rho_{q(x), \Omega_{1}}(\varphi)-\frac{\lambda_{2} t^{q_{2}}}{q_{2}} \rho_{q(x), \Omega_{2}}(\phi) .
\end{aligned}
$$

Let

$$
\begin{aligned}
R_{\alpha} & =M_{2} \rho_{p(x), \Omega_{1}}^{\alpha}(\nabla \varphi)+N_{2} \rho_{p(x), \Omega_{2}}^{\alpha}(\nabla \phi), \\
S_{\lambda_{1}, \lambda_{2}} & =\lambda_{1} \rho_{q(x), \Omega_{1}}(\varphi)+\lambda_{2} \rho_{q(x), \Omega_{2}}(\phi) .
\end{aligned}
$$

Then

$$
J_{\lambda}(t \varphi, t \phi) \leq \frac{t^{\alpha p_{1}}}{\alpha\left(p_{1}\right)^{\alpha}} R_{\alpha}-\frac{t^{q_{2}}}{q_{2}} S_{\lambda_{1}, \lambda_{2}} .
$$

Therefore, we conclude

$$
J_{\lambda}(t \varphi, t \phi)<0,
$$


for $0<t<\sigma^{1 / \alpha p_{1}-q_{2}}$ providing that

$$
0<\sigma<\min \left\{1, \frac{\alpha\left(p_{1}\right)^{\alpha} S_{\lambda_{1}, \lambda_{2}}}{q_{2} R_{\alpha}}\right\}
$$

The proof of Lemma 3.3 is complete.

Proof of Theorem 3.1. From Lemma 3.3, we infer that there exists a ball centered at the origin $\overline{B_{\rho}(0)} \subset E$, such that

$$
\inf _{\partial B_{\rho}(0)} J_{\lambda}>0
$$

Furthermore, by Lemma 3.3, we know that there exists $(\varphi, \omega) \in E$ such that $J_{\lambda}(t \varphi, t \omega)<0$ for $t>0$ small enough. Therefore, considering also inequality (3.14), we obtain that

$$
-\infty<c:=\inf _{B_{\rho}(0)} J_{\lambda}<0
$$

Let us choose $\varepsilon>0$. Then, it follows that

$$
0<\varepsilon \leq \inf _{\partial B_{\rho}(0)} J_{\lambda}-\frac{\inf }{B_{\rho}(0)} J_{\lambda}
$$

Now, if we apply the Ekeland's variational principle [18] to the functional $J_{\lambda}: \overline{B_{\rho}(0)} \rightarrow \mathbb{R}$, it follows that there exists $\left(u_{\varepsilon}, v_{\varepsilon}\right) \in \overline{B_{\rho}(0)}$ such that

$$
\begin{gathered}
J_{\lambda}\left(u_{\varepsilon}, v_{\varepsilon}\right)<\frac{\inf }{B_{\rho}(0)} J_{\lambda}+\varepsilon \\
J_{\lambda}\left(u_{\varepsilon}, v_{\varepsilon}\right)<J_{\lambda}(u, v)+\varepsilon\left\|\left(u-u_{\varepsilon}, v-v_{\varepsilon}\right)\right\|_{E^{\prime}} \quad u_{\varepsilon} \neq u, v \neq v_{\varepsilon} .
\end{gathered}
$$

By the fact that

$$
J_{\lambda}\left(u_{\varepsilon}, v_{\varepsilon}\right)<\frac{\inf }{B_{\rho}(0)} J_{\lambda}+\varepsilon<\inf _{B_{\rho}(0)} J_{\lambda}+\varepsilon<\inf _{\partial B_{\rho}(0)} J_{\lambda}
$$

we can infer that $\left(u_{\varepsilon}, v_{\varepsilon}\right) \in B_{\rho}(0)$.

Now, let us define $\Phi_{\lambda}: \overline{B_{\rho}(0)} \rightarrow \mathbb{R}$ by $\Phi_{\lambda}(u, v)=J_{\lambda}(u, v)+\varepsilon\left\|\left(u-u_{\varepsilon}, v-v_{\varepsilon}\right)\right\|_{E}$. It is not difficult to see that $\left(u_{\varepsilon}, v_{\varepsilon}\right)$ is a minimum point of $\Phi_{\lambda}$, and thus

$$
\frac{\Phi_{\lambda}\left(u_{\varepsilon}+t \cdot \tau, v_{\varepsilon}+t \cdot \sigma\right)-\Phi_{\lambda}\left(u_{\varepsilon}, v_{\varepsilon}\right)}{t} \geq 0
$$


for $t>0$ small enough and any $(\tau, \sigma) \in B_{1}(0)$. By the above expression, we have

$$
\frac{J_{\lambda}\left(u_{\varepsilon}+t \cdot \tau, v_{\varepsilon}+t \cdot \sigma\right)-J_{\lambda}\left(u_{\varepsilon}, v_{\varepsilon}\right)}{t}+\varepsilon\|(\tau, \sigma)\|_{E} \geq 0
$$

Letting $t \rightarrow 0$, we have

$$
\left\langle J_{\lambda}^{\prime}\left(u_{\varepsilon}, v_{\varepsilon}\right),(\tau, \sigma)\right\rangle+\varepsilon\|(\tau, \sigma)\|_{E}>0,
$$

and this implies that $\left\|J_{\lambda}^{\prime}\left(u_{\varepsilon}, v_{\varepsilon}\right)\right\| \leq \varepsilon$. So, we infer that there exists a sequence $\left\{\omega_{n}, v_{n}\right\} \subset B_{\rho}(0)$ such that

$$
J_{\lambda}\left(\omega_{n}, v_{n}\right) \longrightarrow c=\frac{\inf }{B_{\rho}(0)} J_{\lambda}<0, \quad J_{\lambda}^{\prime}\left(\omega_{n}, v_{n}\right) \longrightarrow 0
$$

It is obvious that $\left\{\omega_{n}, v_{n}\right\}$ is bounded in $E$. Therefore, there exists $(\omega, v) \in E$ such that, up to a subsequence, $\left\{\omega_{n}, v_{n}\right\}$ converges weakly to $(\omega, v)$ in $E$ and converges strongly to $\omega_{n} \rightarrow \omega$ in $L^{q(x)}\left(\Omega_{1}\right)$ and $v_{n} \rightarrow v$ in $L^{q(x)}\left(\Omega_{2}\right)$ (Proposition 2.5). Thus $\left\langle J_{\lambda}^{\prime}\left(u_{n}, v_{n}\right),\left(u_{n}-u, v_{n}-\right.\right.$ $v)\rangle \rightarrow 0$ and

$$
\begin{aligned}
& \left\langle J_{\lambda}^{\prime}\left(\omega_{n}, v_{n}\right),\left(\omega_{n}-\omega, v_{n}-v\right)\right\rangle \\
& =M\left(\int_{\Omega_{1}} \frac{\left|\nabla \omega_{n}\right|^{p(x)}}{p(x)} d x\right) \int_{\Omega_{1}}\left|\nabla \omega_{n}\right|^{p(x)-2} \nabla \omega_{n}\left(\nabla \omega_{n}-\nabla \omega\right) d x \\
& \quad+N\left(\int_{\Omega_{2}} \frac{\left|\nabla v_{n}\right|^{p(x)}}{p(x)} d x\right) \int_{\Omega_{2}}\left|\nabla v_{n}\right|^{p(x)-2} \nabla v_{n}\left(\nabla v_{n}-\nabla v\right) d x \\
& \quad-\lambda_{1} \int_{\Omega_{1}}\left|\omega_{n}\right|^{q(x)-2} \omega_{n}\left(\omega_{n}-\omega\right) d x-\lambda_{2} \int_{\Omega_{2}}\left|v_{n}\right|^{q(x)-2} v_{n}\left(v_{n}-v\right) d x \rightarrow 0 .
\end{aligned}
$$

By Proposition 2.1, it follows that

$$
\begin{gathered}
\left.\left|\int_{\Omega_{1}}\right| \omega_{n}\right|^{q(x)-2} \omega_{n}\left(\omega_{n}-\omega\right) d x\left|\leq\left\|\left|\omega_{n}\right|^{q(x)-1}\right\|_{q^{\prime}(x), \Omega_{1}}\left\|\omega_{n}-\omega\right\|_{q(x), \Omega_{1}},\right. \\
\left.\left|\int_{\Omega_{2}}\right| v_{n}\right|^{q(x)-2} v_{n}\left(v_{n}-v\right) d x\left|\leq\left\|\left|v_{n}\right|^{q(x)-1}\right\|_{q^{\prime}(x), \Omega_{2}}\left\|\left|v_{n}-v\right|\right\|_{q(x), \Omega_{2}} .\right.
\end{gathered}
$$

Since $\left\{\omega_{n}\right\}$ converges strongly to $\omega$ in $L^{q(x)}\left(\Omega_{1}\right)$, that is, $\left\|\omega_{n}-\omega\right\|_{q(x), \Omega_{1}} \rightarrow 0$ as $n \rightarrow \infty$, we get

$$
\int_{\Omega_{1}}\left|\omega_{n}\right|^{q(x)-2} \omega_{n}\left(\omega_{n}-\omega\right) d x \longrightarrow 0
$$


and similarly

$$
\int_{\Omega_{2}}\left|v_{n}\right|^{q(x)-2} v_{n}\left(v_{n}-v\right) d x \longrightarrow 0
$$

Hence,

$$
\begin{gathered}
M\left(\int_{\Omega_{1}} \frac{\left|\nabla \omega_{n}\right|^{p(x)}}{p(x)} d x\right) \int_{\Omega_{1}}\left|\nabla \omega_{n}\right|^{p(x)-2} \nabla \omega_{n}\left(\nabla \omega_{n}-\nabla \omega\right) d x \rightarrow 0, \\
N\left(\int_{\Omega_{2}} \frac{\left|\nabla v_{n}\right|^{p(x)}}{p(x)} d x\right) \int_{\Omega_{2}}\left|\nabla v_{n}\right|^{p(x)-2} \nabla v_{n}\left(\nabla v_{n}-\nabla v\right) d x \rightarrow 0 .
\end{gathered}
$$

From $\left(E_{1}\right)$ and $\left(E_{2}\right)$, it follows that

$$
\begin{gathered}
\int_{\Omega_{1}}\left|\nabla \omega_{n}\right|^{p(x)-2} \nabla \omega_{n}\left(\nabla \omega_{n}-\nabla \omega\right) d x \rightarrow 0, \\
\int_{\Omega_{2}}\left|\nabla v_{n}\right|^{p(x)-2} \nabla v_{n}\left(\nabla v_{n}-\nabla v\right) d x \longrightarrow 0 .
\end{gathered}
$$

Eventually, by [19, Theorem 3.1], we get that $\left\{\omega_{n}, v_{n}\right\}$ converges strongly to $(\omega, v)$ in $E$, so we conclude that $(\omega, v)$ is a nontrivial weak solution for problem $(\mathrm{P})$. The proof of Theorem 3.1 is complete.

\section{Acknowledgments}

The authors would like to thank the referees for their many helpful suggestions and corrections, which improve the presentation of this paper. This research was supported by DUBAP grant no. 10-FF-15, Dicle University, Turkey.

\section{References}

[1] M. Růžička, Electrorheological Fluids: Modeling and Mathematical Theory, vol. 1748 of Lecture Notes in Mathematics, Springer, Berlin, Germany, 2000.

[2] V. V. Zhikov, "Averaging of functionals of the calculus of variations and elasticity theory," Mathematics of the USSR-Izvestiya, vol. 9, no. 4, pp. 33-66, 1986.

[3] S. N. Antontsev and S. I. Shmarev, "A model porous medium equation with variable exponent of nonlinearity: existence, uniqueness and localization properties of solutions," Nonlinear Analysis: Theory, Methods \& Applications, vol. 60, no. 3, pp. 515-545, 2005.

[4] O. A. Ladyzhenskaya and N. N. Ural'tseva, Linear and Quasilinear Elliptic Equations, Academic Press, New York, NY, USA, 1968.

[5] K. Pflüger, "Nonlinear transmission problems in bounded domains of $\mathbb{R}^{n}$," Applicable Analysis, vol. 62, no. 3-4, pp. 391-403, 1996.

[6] T. F. Ma and J. E. Muñoz Rivera, "Positive solutions for a nonlinear nonlocal elliptic transmission problem," Applied Mathematics Letters, vol. 16, no. 2, pp. 243-248, 2003.

[7] A. Marzocchi, J. E. Muñoz Rivera, and M. G. Naso, "Transmission problem in thermoelasticity with symmetry," IMA Journal of Applied Mathematics, vol. 68, no. 1, pp. 23-46, 2003. 
[8] J. E. Muñoz Rivera and H. P. Oquendo, "The transmission problem of viscoelastic waves," Acta Applicandae Mathematicae, vol. 62, no. 1, pp. 1-21, 2000.

[9] J. Y. Park, J. J. Bae, and I. H. Jung, “Uniform decay of solution for wave equation of Kirchhoff type with nonlinear boundary damping and memory term," Nonlinear Analysis: Theory, Methods $\mathcal{E}$ Applications, vol. 50, no. 7, pp. 871-884, 2002.

[10] C. O. Alves and F. J. S. A. Corrêa, "On existence of solutions for a class of problem involving a nonlinear operator," Communications on Applied Nonlinear Analysis, vol. 8, no. 2, pp. 43-56, 2001.

[11] F. J. S. A. Corrêa and G. M. Figueiredo, "On an elliptic equation of $p$-Kirchhoff type via variational methods," Bulletin of the Australian Mathematical Society, vol. 74, no. 2, pp. 263-277, 2006.

[12] G. Dai and R. Hao, "Existence of solutions for a $p(x)$-Kirchhoff-type equation," Journal of Mathematical Analysis and Applications, vol. 359, no. 1, pp. 275-284, 2009.

[13] G. Dai and D. Liu, "Infinitely many positive solutions for a $p(x)$-Kirchhoff-type equation," Journal of Mathematical Analysis and Applications, vol. 359, no. 2, pp. 704-710, 2009.

[14] X. Fan and D. Zhao, "On the spaces $L^{p(x)}(\Omega)$ and $W^{m, p(x)}(\Omega)$," Journal of Mathematical Analysis and Applications, vol. 263, no. 2, pp. 424-446, 2001.

[15] O. Kováčik and J. Rákosník, “On spaces $L^{p(x)}$ and $W^{k, p(x)}$, " Czechoslovak Mathematical Journal, vol. 41(116), no. 4, pp. 592-618, 1991.

[16] X. Fan, J. Shen, and D. Zhao, "Sobolev embedding theorems for spaces $W^{k, p(\cdot)}(\Omega)$," Journal of Mathematical Analysis and Applications, vol. 262, no. 2, pp. 749-760, 2001.

[17] S.-G. Deng, "Positive solutions for Robin problem involving the $p(x)$-Laplacian," Journal of Mathematical Analysis and Applications, vol. 360, no. 2, pp. 548-560, 2009.

[18] I. Ekeland, "On the variational principle," Journal of Mathematical Analysis and Applications, vol. 47, pp. 324-353, 1974.

[19] X.-L. Fan and Q.-H. Zhang, "Existence of solutions for $p(x)$-Laplacian Dirichlet problem," Nonlinear Analysis: Theory, Methods E Applications, vol. 52, no. 8, pp. 1843-1852, 2003. 


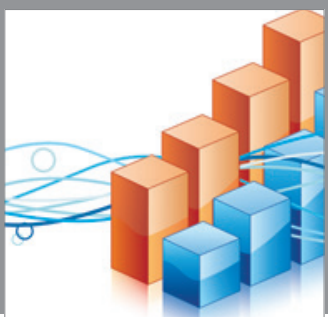

Advances in

Operations Research

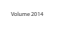

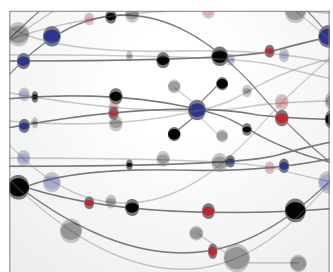

\section{The Scientific} World Journal
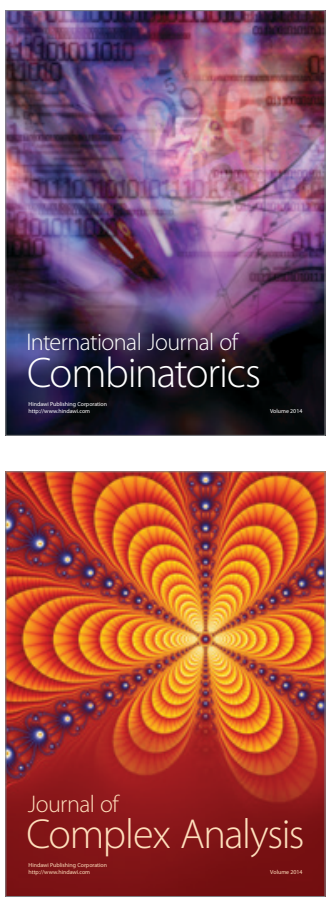

International Journal of

Mathematics and

Mathematical

Sciences
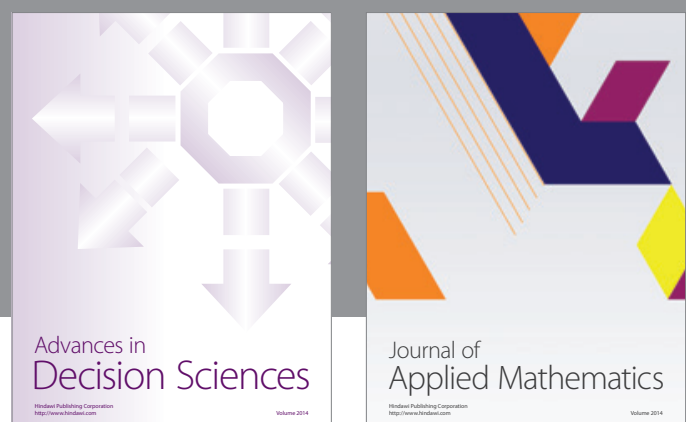

Journal of

Applied Mathematics
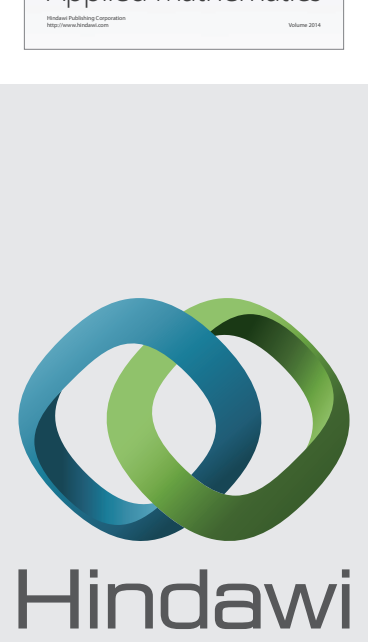

Submit your manuscripts at http://www.hindawi.com
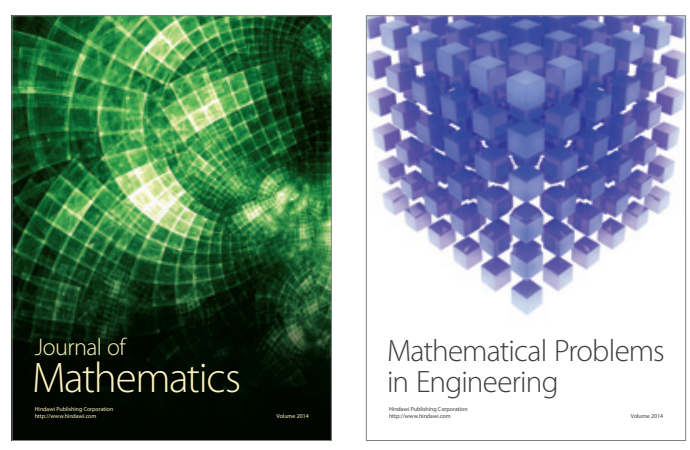

Mathematical Problems in Engineering
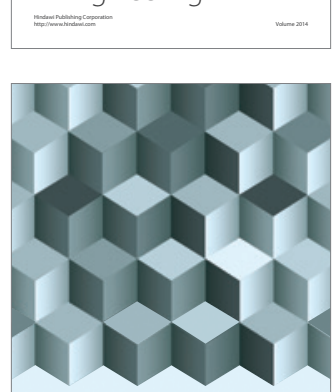

Journal of

Function Spaces
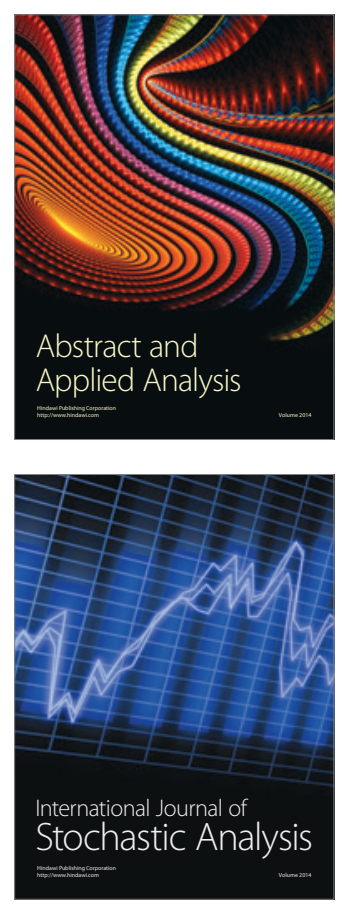

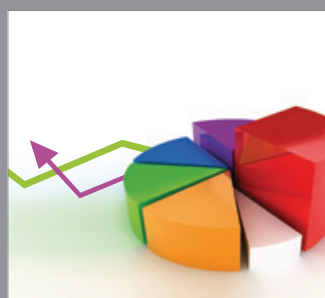

ournal of

Probability and Statistics

Promensencen
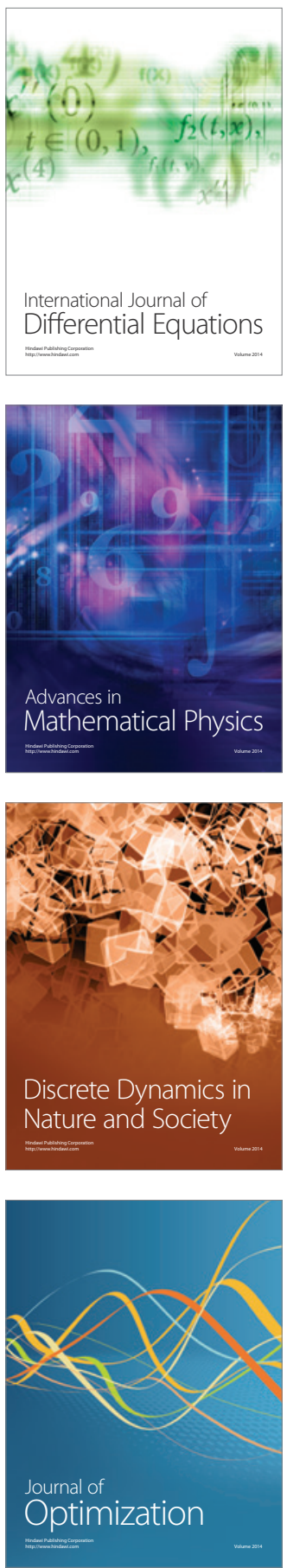